\title{
Symptoms and signs of lower gastrointestinal disease
}

Mica Schofield, BSc University of East Anglia Medical School, mica.schofield@hotmail.co.uk

*Anton Emmanuel, MD FRCP GI Physiology Unit, University College Hospital, Grafton Way, London WC1E 6DB, Tel: 0203447 0697, a.emmanuel@ucl.ac.uk

*author for correspondence

Keywords: diarrhoea, constipation, abdominal pain, faecal incontinence

\section{Key points:}

- Common lower gut symptoms most often have a benign cause, but eliciting alarm symptoms is key to exclude the possibility of organic disease.

- Diarrhoea: determining the characteristics of the stool as either secretory, osmotic, inflammatory or dysmotility-related helps define the likeliest causes of the symptom.

- Constipation: symptoms can help with a pathogenetic classification based around transit, evacuation difficulty, a combination of these two, or irritable bowel syndrome.

- Faecal incontinence: a common symptom that is often under-reported by patients and which benefits from taking an approach to determine whether the cause is due to either anal sphincter or rectum.

- Rectal bleeding: whilst most often due to benign local causes, it is essential to consider the possibility of colonic diseases which may be amenable to treatment.

\footnotetext{
Abstract:

This chapter provides a physiological basis for the understanding of a practical approach to patients presenting with the common lower intestinal complaints. These symptoms arise from disturbances of gut motility (diarrhoea or constipation), coordination and function of the anorectum (evacuation difficulty and faecal incontinence)and structural deficits of the hindgut (rectal bleeding). Abdominal pain is a common presentation and this can be related to physiological or anatomical deficits in the gut. This chapter will describe the clinical assessment of such patients in order to discern the likeliest diagnosis of abdominal pain.
} 


\section{Diarrhoea}

Stool consistency is determined by the interplay of water secretion and absorption within the bowel. When water secretion largely exceeds absorption, watery stools are produced. Diarrhoea is objectively defined as a stool weight of over 200g within a 24-hour period. However, since this is not a practical diagnostic feature in clinical practice, three or more loose or liquid stools (Bristol stool form 5 or above) per day is used (1). Frequency and consistency of normal bowel movements varies widely, and it is important to elicit whether there has been a change from the norm.

Type 1
Type 2
Type 3
Type 5
Type 6

Acute diarrhoea (typically defined as lasting less than 4 weeks) (1) is likely to be due to infection. Questioning regarding recent foreign travel and food consumption may elicit the source, although it is important to consider acute diarrhoea as the initial presentation of a chronic cause. Clinical suspicion of certain infections may require prompt notification to Public Health England to prevent possible epidemics. The most common of these is food poisoning.

Chronic diarrhoea (symptoms over 4 weeks) is a common presentation, affecting up to $5 \%$ of Western populations (1). Although an infective cause (e.g. Giardia) is possible, more probable differentials exist. It is helpful to think of these potential causes in terms of the underlying pathophysiology, which can be categorised into three main groups. History taking should include stool characteristics as well as associated features (see Box 1). It is important to remember that medications can also cause diarrhoea.

\section{Box 1: Associated features}

- Steatorrhoea (pale stools which are difficult to flush) indicates small bowel or pancreatic pathology

- Abdominal pain relieved by bowel movement suggests irritable bowel syndrome

- Weight loss and nocturnal diarrhoea indicate an organic cause - must be investigated

- Lightheadedness, tachycardia and low urine output may be secondary to dehydration

- Blood in stool indicates inflammatory bowel disease

(IBD) or infectious colitis

- Oral ulceration and perianal disease is seen in 
Clinical features: Watery, large volume and frequent bowel movements. Often, there is no associated pain. Diarrhoea does not settle with periods of fasting.

Pathophysiology: Increased $\mathrm{Cl}^{-}$secretion and impaired $\mathrm{Na}^{+}$transport due to activation of transport processes within the enterocyte (often caused by a peptide or toxin). This leads to increased fluid secretion and decreased absorption, causing watery stools.

\section{Examples:}

- Toxins e.g. E Coli and Vibrio cholerae

- Hormones e.g. carcinoid tumours and vasoactive intestinal peptide

- Bile acid malabsorption (increased levels of bile salts in colon stimulates electrolyte secretion)

\section{Osmotic diarrhoea}

Clinical features: Bulky or "pasty" stool which settles with fasting or avoidance of causative substance. Steatorrhoeatype stool may occur: pale stools which are difficult to flush.

Pathophysiology: Ingestion of poorly-absorbed substances which are osmotically-active (often magnesium salts and sugars such as mannitol). This causes fluid retention within the bowel lumen.

\section{Examples:}

- Malabsorption disorders: Coeliac disease, bowel resection and bacterial overgrowth

- Maldigestion disorders: lactase deficiency and pancreatic insufficiency (as seen in chronic pancreatitis and cystic fibrosis)

- Osmotic laxative overuse

\section{Motility-related diarrhoea}

Clinical features: Associated with cramping abdominal pain. Wide variety in stool consistency. Episodic constipation may occur.

Pathophysiology: Variable rate of transit through the digestive tract related to gut nerve or muscle dysfunction. Decreased contact time between intestinal contents and the mucosa prevents sufficient reabsorption.

\section{Examples:}

- IBS

- Stimulant laxative overuse

- Intestinal resection

In patients with inflammatory bowel disease (IBD), the underlying pathophysiology is likely to be due to a combination of mechanisms and therefore may be difficult to treat. Patients may complain of blood in the stool and may have additional symptoms such as fever, cramping abdominal pain and tenesmus.

All patients should be asked about symptoms of dehydration, a common complication of diarrhoea. Inability to retain oral fluids or signs of severe dehydration require hospital admission. 
Box 2: Alarm features (2)

- $\quad$ Age $>50$

- Short history of symptoms

- Male sex

- Unintentional weight loss

- Nocturnal symptoms

- Anaemia

- Rectal bleeding

- Abdominal mass

- Recent antibiotic use

- Family history of bowel/ovarian cancer

\section{Constipation}

Constipation is a frequent complaint, experienced by 1 in 7 adults and 1 in 3 children (depending on the specific criteria used) (3). In the developed world, women are more commonly affected than men, and prevalence increases with age. Over one-third of women experience constipation during pregnancy, especially in the first two trimesters. The costs of constipation are great - data from the UK for 2015 suggests that unplanned hospital admission costs for constipation were f145million, laxative costs were f101million and constipation causes a reduction of 2.4 days per month of work/school productivity (3)

The symptoms of constipation are multiple, but the standard for classification in clinical trials is set by the Rome IV criteria, summarised in Box 3 . In clinical practice, these criteria are of little value given their complexity of application. A more practical classification depends on the individual's perception of normal bowel habit and refers to either infrequency or difficulty with defaecation. Patients with self-reported constipation are most bothered by symptoms of straining, abdominal discomfort and a sensation of incomplete evacuation. Describing stool consistence can be difficult for some patients, and the Bristol Stool Form Scale (types 1 and 2) can be helpful.

\section{Box 3: Rome IV criteria for functional constipation}

1. Must include 2 or more of the following:

a. Straining during more than one-fourth $(25 \%)$ of defecations

b. Lumpy or hard stools (Bristol stool form scale 1-2) more than one-fourth $(25 \%)$ of defecations

c. Sensation of incomplete evacuation more than one-fourth $(25 \%)$ of defecations

d. Sensation of anorectal obstruction/blockage more than one-fourth $(25 \%)$ of defecations

e. Manual manoeuvres to facilitate more than one-fourth (25\%) of defecations (e.g. digital evacuation, support of the pelvic floor)

f. Fewer than 3 spontaneous bowel movements per week

2. Loose stools are rarely present without the use of laxatives

3. Insufficient criteria for irritable bowel syndrome

It is important to classify constipation into primary and secondary causes due to differences in management. Secondary constipation may be a consequence of systemic conditions, medications or structural abnormalities within the gastrointestinal tract. Specific causes of secondary constipation are shown in Box 4. Primary constipation is related to colonic or anal dysfunction and can be characterised pathophysiologically into four main categories:

i) slow transit,

ii) defaecation disorder,

iii) a combination of i) and ii)

iv) normal transit.

\section{Slow transit constipation}

This is typically a disorder of young women who often give a history of constipation since childhood. The cause may relate to colonic neuromuscular dysfunction. The reduced motility (in both colon and small bowel) leads to prolonged 
transit time and hence infrequent urge to void and a more dehydrated small volume stool. Abdominal pain and bloating are also features.

\section{Defaecation disorder}

This can arise from either a structural or physiological disorder. The mechanical causes include a rectocele (anterior bulge of the rectum in to the posterior wall of the vagina), rectal prolapse or intussusception (of the recto-anal unit). The latter may be related to the physiological component which can be related to failure of relaxation of the pelvic floor muscles or paradoxical contraction of the internal anal sphincter ("anismus") during attempted voiding. These pelvic floor factors are often contributed to by ineffective propulsion from the abdominal musculature. Patient presents with an inability to void their rectum despite urge, so they may strain despite having soft stools and have a sense of incomplete emptying. They may need to adopt uncomfortable positions on the toilet to void and may use digital manoeuvres to aid defaecation.

\section{Normal transit constipation}

This is a term given to the presence of constipation symptoms when there is no demonstrable delay in transit or pelvic floor dysfunction. The symptoms can be just as intrusive for the patient as with any of the other pathophysiological subtypes.

A small proportion of patients presenting with constipation will have a megacolon. This is a condition that can be either inherited or acquired and results in dilatation of the rectum and possibly more proximal colon. The dilatation is associated with loss of tone and slowed transit, so patients present with both features of slow transit and difficult evacuation.

\section{Assessment}

The clinical history is key to identify possible alarm features (see box 2) and secondary causes of constipation. With regard to primary causes, whilst there is a great deal of symptom overlap, history taking may point towards one form of pathophysiology more than another (as outlined above).

Abdominal examination is essential to identify potential secondary causes (especially endocrine or neurological). An abdominal faecal mass may suggest a megacolon. A digital rectal examination is important to exclude faecal impaction (remembering that a small amount of soft stool is present in the normal rectum) as well as identify any possible colonic lesion causing the problem. Equally important, careful examination with the finger may help identify paradoxical pelvic floor or sphincter contraction during attempted voiding. Identifying haemorrhoids which have complicated chronic constipation or the presence of anal fissures or a rectal prolapse are other key aspects of the perineal examination.

\section{Box 4: Secondary causes of constipation}

Medications: opiates, ferrous supplements, tricyclic antidepressants, diuretics, antipsychotics

Intrinsic colonic pathology: colorectal cancer, diverticular disease

Metabolic/endocrine: hypercalcaemia, coeliac disease, hypothyroidism, hypocalcaemia

Neurological: spinal cord injury, multiple sclerosis, Parkinson's disease

Psychological: depression, anorexia nervosa, bulimia, affective disorders, abuse

\section{Faecal incontinence}

Faecal incontinence is the involuntary passage of stool (and flatus) from the back passage. It affects up to $10 \%$ of the adult population, increasing in prevalence with age (4). However, this is likely to be an underestimate due to the 


\section{Box 6: Physiology of continence}

Arrival of faeces or flatus into the rectum is detected by stretch receptors and at low volumes is accommodated. At critical ill volume, new content causes relaxation of the internal anal sphincter.

If defecation is inconvenient, voluntary contraction of the external anal sphincter occurs. This allows anal pressure to remain higher than rectal pressure and delays evacuation.

perceived stigma attached to symptoms. Patients experience a significant decrease in their quality of life, leading to low mood and social isolation. It is important to be aware that faecal incontinence is a symptom, not a diagnosis and often has a complex aetiology with many contributory factors (see box 5).

\section{Factors important for continence}

- $\quad$ Function of the internal (involuntary) and external (voluntary) anal sphincters

- Neurological motor control over anal canal and pelvic floor muscles

- Neurological sensory function to sense contact at an appropriate volume and timing

- Anal cushions (as mechanical barrier)

- Stool consistency

- Rectal compliance (to accommodate stool content delivered by colon)

Defects in any one or more of the above can cause faecal incontinence. Common causes of incontinence are cited in box 5 .

Faecal incontinence is clinically classified into two symptom types:

\section{Urge incontinence}

- Sudden need to defecate with insufficient warning to reach a toilet

- Causes: diarrhoea, or weakness/defects of the external anal sphincter, or loss of normal anorectal sensation

- The symptom of urgency itself (without incontinence) is distressing and limits quality of life

\section{Passive incontinence}

- Loss of faeces or flatus without sensory awareness, resulting in soiling of underwear or insensate loss of wind

- Causes: diarrhoea, weakness/defects of the internal anal sphincter, or damage to the anal cushions, or loss of rectal compliance

Symptom assessment should include classifying the kind of incontinence (as above) as well as the frequency and consistency of stool passed. It is also important to enquire about obstetric, neurological and surgical history to determine potential aetiology. The history needs to be sensitively taken to address the potential embarrassment and hesitance of the patient. In addition, the impact on the patient's lifestyle needs to be addressed - dietary patterns, lifestyle adjustments and medications used. Symptom burden and quality of life impact can be supplemented through use of severity scales such as the Cleveland Clinic or St Mark's scores.

General examination may reveal evidence of abdominal scars or neurological disease. Perianal inspection may reveal evidence of obstetric trauma, significant haemorrhoids or rectal prolapse (which can present at any age). Digital rectal examination is essential to assess anal tone both at rest (internal anal sphincter function) and on contraction (external anal sphincter). It may also aid diagnosis of chronic constipation (which may be causing overflow diarrhoea).

\section{Rectal bleeding}

The passage of blood per rectum ("haematochezia") is common, affecting approximately $10 \%$ of UK adults at any one time. Although most often caused by benign conditions of the colon or anus, serious conditions such as malignancy and IBD must be excluded. Haemodynamic instability associated with large volume rectal bleeding should provoke consideration of an upper $\mathrm{Gl}$ source.

It is important to be precise about the exact symptom a patient is describing. Details of whether blood is seen mixed in the stool, is present in the toilet bowl or only on wiping may help localise the bleeding source: blood seen only on wiping post-defecation is likely to be due to anal causes (fissure or haemorrhoids), whereas blood mixed in the stool may originate from higher up in the GI tract. 
- Duration and frequency of bleeding

- Acute, intermittent or chronic?

- Volume of blood

- Streaks or frank blood? Passage of clots suggest heavier bleeding.

- Colour of blood

- Bright red blood is usually from the left colon or distal; dark blood is typically due to a more proximal lesion.

- Change in bowel habit?

- Associated symptoms

- Haematemesis suggests upper GI cause; passing mucus may be associated with IBD or rectal adenomas; dizziness and syncope may indicate large volume blood loss; associated pain and fevers raises the possibility of diverticular disease

- Other alarm features

- Weight loss, abdominal/rectal mass, personal or family history of IBD/bowel cancer and anaemia

- Past medical history

○ Including IBD, coagulopathies and conditions requiring anticoagulation

- Drug history

- Especially anticoagulants and conditions requiring anticoagulation

- Travel history

Age is important in determining the likeliest differentials of rectal bleeding: new rectal bleeding in patients over 50 should raise the possibility of malignancy or diverticular disease and there should be consideration of urgent referral for investigation. Alongside abdominal examination, all patients require a digital rectal examination to exclude a palpable rectal mass. It is important to remember that discovery of haemorrhoids or an anal fissure on examination does not exclude other causes of rectal bleeding.

\section{Box 7: Common causes of rectal bleeding}

- Haemorrhoids

- Anal fissure

- Diverticular disease

- Colorectal/anal malignancy

- Inflammatory bowel disease

- Colonic polyps

- Ischaemic colitis

- Infection

\section{Abdominal pain}

Abdominal pain is a common presentation to both primary and secondary care services. Annually, abdominal pain is responsible for 300,000 emergency admissions and a further 90,000 non-urgent hospital admissions in England alone (5). The differentials of abdominal pain are vast and may arise from a variety of different body systems (see Table 1); due to this, history taking is of the upmost importance to ascertain the most likely diagnosis. Despite investigation, many patients with chronic abdominal pain (lasting over six months) have no evidence of organic pathology.

The character of the pain aids identification of the underlying pathology. Visceral pain is a consequence of distension, inflammation or ischaemia of viscera or the capsule in which they are encased. This pain is characteristically described as a diffuse in location and dull in character. The general pain location correlates the embryological origin of the affected organ. Due to association with the autonomic nervous system, pain may be associated with autonomic features such as sweating, pallor and changes in vital signs. Conversely, somatic pain arises from irritation to the parietal peritoneum which lines the abdominal and pelvic cavities. This produces well-localised, sharp pain which the patient can accurately point to using one finger. 
- Location

- Well-localised or diffuse? Epigastric pain is related to foregut structures; mid-abdominal pain relates to midgut structures; suprapubic pain derives from hindgut pathology

- Onset

- Sudden onset pain may be secondary to infarction, rupture or perforation; gradual pain may arise from chronic inflammation or malignancy

- Character

- Dull ache (visceral pain) or sharp, stabbing pain (somatic pain)? Wave-like colicky pain suggests intestinal obstruction or renal/biliary colic

- Radiation

- Shoulder tip pain due to diaphragmatic irritation; renal colic radiates to the groin; back pain may be referred from an abdominal aortic aneurysm or pancreatic conditions

- Exacerbating and alleviating factors

- Correlation with meals may indicate peptic ulcer disease, cholecystitis or small bowel pathology; pain

\begin{tabular}{|c|c|c|}
\hline \multicolumn{2}{|c|}{ Table 1: Common differential diagnoses of abdominal } \\
pain
\end{tabular}
alleviated by defaecation suggests rectal pathology or irritable bowel syndrome; pain worsened by movement may be peritonitis

- $\quad$ Associated symptoms

- Presence of vomiting suggests gastroenteritis, appendicitis, pancreatitis or small bowel obstruction; respiratory symptoms may suggest lower lobe pneumonia; rectal bleeding may indicate IBD

- $\quad$ Last menstrual period (in females of child-bearing age)

- Mid-cycle pain suggests mittelschmerz; missed periods raises the suspicion of ectopic pregnancy

- Past medical and surgical history

- Adhesions from previous abdominal surgery may lead to small bowel obstruction

\section{- Social history}

- Alcohol excess is a known risk factor for pancreatitis; there is an association between smoking and development/severity of Crohn's disease

Examination should include general inspection, looking for signs of cachexia. Peripheral stigmata of liver disease such as spider naevi, bruising, palmar erythema and jaundice should increase speculation of liver cirrhosis. Abdominal examination should include palpation of all areas (classically the abdomen being divided into a $3 \times 3$ grid) beginning away from the affected area. Tenderness, guarding and rebound tenderness should be noted. Palpable masses require urgent investigation. It is important to note that even large volume ascites may be painless. Hyperactive bowel sounds suggest intestinal obstruction whereas reduced/absent bowel sounds may be present in peritonitis or paralytic ileus. Inspection of the inguinal area and external genitalia are vital to exclude hernias and testicular torsion respectively.

In light of the differential diagnoses (Table 1), a digital rectal examination is an important component of the examination in the patient with abdominal pain. Once consent is granted, a pregnancy test should be performed in all women of childbearing age who present with abdominal pain, to exclude gynaecological emergencies such as ruptured ectopic pregnancy. 
1. A 36-year-old man reports 3-months of opening her bowels 5-6 times a day. The stools are watery and occasionally pale in colour. There is occasional nocturnal diarrhoea. He has type 2 diabetes and takes a sulphonylurea. He had a cholecystectomy 2 years previously. There has been no weight loss and his blood tests including tissue transglutaminase are normal. Which of the following are the likeliest causes of her diarrhoea:
a. bile acid diarrhoea
b. diverticular disease
c. drug-induced diarrhoea
d. Helicobacter pylori infection
e. small intestinal bacterial overgrowth

2. A 27-year-old woman gives a 5-year history of abdominal bloating, weight gain and constipation. The pain is eased by bowel opening, which occurs every 5-7 days. Stool consistency varies from pellet-like to semi-formed. She strains at stool on every occasion. What has made her consult now is the presence of anal discomfort with no bleeding. Which of the following is true with regards to rectal examination:
a. a tear in the anal skin is normal with chronic straining, and unlikely to be a cause of her pain
b. contraction of the internal anal sphincter is normal on bearing down
c. low resting anal pressure excludes an anal fissure
d. rectal prolapse is possible despite her young age
e. the presence of stool in the rectum confirms the diagnosis of faecal impaction

3. A 53 year old woman presents via the Emergency Department having had 4 stereotyped episodes in the previous 8 months. She develops acute abdominal pain with vomiting and on each occasion this settles with 24 hours after which feels well. She does not pass stools for a day or two and tends to avoid meals for that period. On this occasion, the pain has persisted for 36 hours. Her weight is steady and her ondly previous hospitalisation was for peritonitis complicating acute appendicitis aged 13. On examination, she is uncomfortable and afebrile. Her abdomen is tender with active bowel sounds. What is the likeliest diagnosis:
a. diverticular disease
b. endometriosis
c. inflammatory bowel disease
d. irritable bowel syndrome
e. subacute intestinal obstruction

\section{References}

1. Arasaradnam R, Brown S, Forbes A, Fox M, Hungin P, Kelman L et al. Guidelines for the investigation of chronic diarrhoea in adults: British Society of Gastroenterology, 3rd edition. Gut [Internet]. 2018;67(8):1380-1399. Available from: https://gut.bmj.com/content/67/8/1380

2. Spiller R, Aziz Q, Creed F, Emmanuel A, Houghton L, Hungin P et al. Guidelines on the irritable bowel syndrome: mechanisms and practical management. Gut [Internet]. 2007;56(12):1770-1798. Available from: https://www.bsg.org.uk/asset/C5702D38-4D06-4EE3AE34EA7746ACAA5B

3. Coloplast. The Cost of Constipation report [Internet]. Coloplast; 2016. Available at: https://www.coloplast.co.uk/Global/UK/Continence/Cost of Constipation Report FINAL.pdf

4. Duelund-Jakobsen J, Worsoe J, Lundby L, Christensen P, Krogh K. Management of patients with faecal incontinence. Therapeutic Advances in Gastroenterology [Internet]. 2015;9(1):86-97. Available from: http://journals.sagepub.com/doi/pdf/10.1177/1756283X15614516

5. Royal College of Surgeons of England. Commissioning guide: Emergency general surgery (acute abdominal pain) [Internet]. Royal College of Surgeons of England; 2014 [cited 3 November 2018]. Available from: https://www.rcseng.ac.uk/_media/files/rcs/standards-and-research/commissioning/commissioning-guide--egs-published-v3.pdf 